\title{
Lidil
}

Revue de linguistique et de didactique des langues

\section{Utilisation des pronoms personnels en français écrit par des enfants sourds bilingues}

Un parcours spécifique d'apprentissage?

Nathalie Niederberger et loanna Berthoud-Papandropoulou

\section{OpenEdition}

\section{Journals}

Édition électronique

URL : http://journals.openedition.org/lidil/623

DOI : $10.4000 /$ lidil.623

ISSN : 1960-6052

Éditeur

UGA Éditions/Université Grenoble Alpes

Édition imprimée

Date de publication : 1 décembre 2004

Pagination : 27-38

ISBN : 2-914176-11-2

ISSN : $1146-6480$

Référence électronique

Nathalie Niederberger et loanna Berthoud-Papandropoulou, «Utilisation des pronoms personnels en français écrit par des enfants sourds bilingues », Lidil [En ligne], 30 | 2004, mis en ligne le 29 janvier 2008, consulté le 01 mai 2019. URL : http://journals.openedition.org/lidil/623 ; DOI : 10.4000/lidil.623 


\title{
UTILISATION DES PRONOMS PERSONNELS EN FRANÇAIS ÉCRIT PAR DES ENFANTS SOURDS BILINGUES : UN PARCOURS SPÉCIFIQUE D'APPRENTISSAGE?
}

\author{
Nathalie NIEDERBERGER * \\ IOANIA BERTHOUD-PAPANDROPOULOU **
}

Plusieurs études ont montré que l'apprentissage de l'écrit est problématique pour les enfants sourds profonds qui rencontrent des difficultés considérables, en particulier dans le domaine de la morphosyntaxe (Hage, 1994; Jacq, Tuller, et Fuet, 1999; Tuller, 2000). Parmi les erreurs fréquemment observées, on relève l'ordre des mots inapproprié, des marques morphologiques incorrectes et des omissions de déterminants et de pronoms (Dubuisson et Bastien, 1998; Lepot-Froment et Clerebaut, 1996). Ces erreurs sont souvent attribuées aux compétences insuffisantes dans la langue orale (Leybaert et Alegria, 1986). Or on peut se demander si une connaissance limitée de l'oral ne présenterait pas paradoxalement des avantages pour certains aspects de l'apprentissage de l'écrit. Plus particulièrement, les enfants sourds ne commettraient pas certaines erreurs fréquemment observées chez les enfants entendants, qui au début ne différencient pas dans l'écrit le pluriel du singulier lorsque cela ne s'entend pas à l'oral (ex: il-ils; mange-mangent). Notre étude, centrée sur l'emploi des pronoms personnels, vise à répondre à cette question et à investiguer la possibilité pour les enfants sourds

* Psycholinguistique, Université de Genève et Fonds national suisse pour la recherche scientifique.

** Psycholinguistique, Université de Genève. 
d'un parcours d'apprentissage de l'écrit en partie différent de celui des enfants entendants (Niederberger et BerthoudPapandropoulou, 2002), comme le suggère une étude portant sur l'apprentissage de l'orthographe anglaise par les enfants sourds révélant un pattern d'erreurs très différent de celui habituellement observé chez les petits anglophones (Padden, 1993).

Nous supposons que les enfants sourds qui utilisent dès les premières années à la fois la langue française et la Langue des Signes Française (LSF) dans les cadres familial et scolaire développent certains aspects de la langue écrite alors qu'ils ne maitrisent pas (encore) ces mêmes aspects à l'oral. Ce serait notamment le cas pour les pronoms personnels, dont nous allons étudier l'emploi, à l'oral et à l'écrit, par des enfants sourds suivant une scolarité bilingue en LSF et en français oral et écrit ${ }^{1}$. La connaissance de cette langue gestuelle et la compétence métalinguistique de ces sujets bilingues seront envisagées comme des outils alternatifs dans l'apprentissage de l'écrit, hypothèse défendue par plusieurs groupes de chercheurs (Chamberlain et Mayberry, 2000; Prinz et Strong, 1998).

\section{Méthode}

\section{Populatation}

Onze sujets sourds bilingues LSF/français d'un même établissement scolaire de Genève, quatre filles et sept garçons, ont été interrogés; ils sont âgés de $8 ; 5$ à $14 ; 10$ ans (âge moyen: $11 ; 4)$. Ils présentent tous une surdité sévère ou profonde, avec des degrés variables de récupération auditive avec appareillage. Exposés de manière régulière à la LSF et au français avant l'âge de 5 ans lors d'activités pré-scolaires et dans leur cadre familial (la plupart des parents ont suivi des cours de LSF), ils suivent actuellement à la journée un enseignement

1. Cette recherche a été réalisée dans le contexte d'un projet plus large de thèse de doctorat étudiant les relations entre capacités langagières développées en LSF et en français chez les enfants sourds bilingues de Suisse romande (Niederberger, 2004). 
bilingue LSF/français oral et écrit dans une structure publique comprenant enseignants sourds et entendants ${ }^{2}$.

Quarante enfants entendants, 21 filles et 19 garçons, constituent le groupe contrôle. La moitié d'entre eux sont âgés de 7;1 à 7;11 ans (âge moyen: 7;7) et fréquentent une classe de $2^{\text {e }}$ primaire (groupe $2 \mathrm{P}$ ). Les vingt autres, âgés de $9 ; 0$ à 10;1 ans (âge moyen: 9;6), fréquentent une classe de $4^{e}$ primaire (groupe $4 \mathrm{P}$ ). Ils proviennent de la même école à Genève. Onze d'entre eux au moins sont également bilingues et utilisent de manière régulière une autre langue que le français (portugais, italien, suisse allemand ou albanais).

Tous les milieux socio-économiques sont représentés, aussi bien dans le groupe des enfants sourds que dans le groupe contrôle.

\section{Matériel}

Deux épreuves ont été constituées, l'une en modalité orale et l'autre en modalité écrite, afin de susciter la production de pronoms personnels dans des contextes appropriés. Elles sont composées chacune de dix items précédés d'un exemple. Chaque item consiste en une ou deux images accompagnées d'une courte phrase, présentée oralement ou par écrit selon la modalité et débouchant sur une question à laquelle le sujet doit répondre par oral ou par écrit (exemple item 1) ou sur une seconde phrase à compléter (exemple item 2 ).

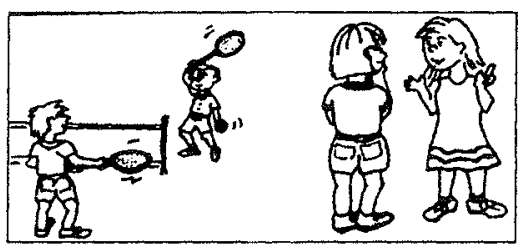

Item I

Les garçons jouent au tennis. Que font les filles?

(Pronom sujet féminin pluriel (elles) + Verbe)

2. Voir Niederberger (sous presse) pour une description détaillée de ce type particulier de programmes scolaires bilingues. 


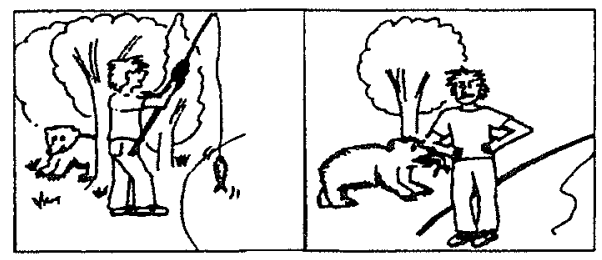

Item 2

Le garçon a pêché un poisson. L'ours...

(Pronom objet masculin singulier $(\mathrm{le})+$ Verbe)

Le matériel vise à induire une production impliquant l'utilisation d'un pronom bien que la consigne donnée au sujet ne le demande pas explicitement. Les pronoms attendus varient d'un point de vue morphologique (masculin/féminin, singulier/pluriel) et syntaxique (sujet/objet). Rappelons que, en français, pour le pronom sujet aussi bien masculin que féminin, le pluriel se différencie du singulier en modalité écrite mais pas à l'oral (il/ils et elle/elles).

\section{Procédure}

Les épreuves ont été présentées lors de deux sessions différentes, la version écrite lors de la première session et la version orale lors de la deuxième session, afin de ne pas exposer d'emblée les enfants sourds à la tâche la plus difficile pour eux.

En modalité écrite, l'expérimentatrice donnait au sujet la consigne suivante, en lui montrant le matériel:

Sur chaque feuille, il y a des images, et sous les images, il y a un petit texte avec une question ou une phrase qu'il faut terminer. Alors toi tu vas bien regarder les images, ensuite tu lis le texte, et tu réponds à la question ou tu continues la phrase.

En modalité orale, la consigne était similaire:

Sur chaque feuille, il y a des images. Moi je vais te dire un petit texte et tu vas bien écouter. A chaque fois à la fin il y aura une question et toi tu dois répondre, ou une phrase que je commence et toi tu dois la terminer.

Les productions orales des sujets ont été enregistrées et filmées puis transcrites ${ }^{3}$.

3. Nous tenons à remercier les étudiantes qui ont participé à ce travail dans le cadre de leur deuxième cycle de Psychologie à l'Université de Genève. 


\section{Codage}

Les réponses des sujets ont été évaluées sur les pronoms attendus: un point a été attribué par pronom approprié. Le score maximum par épreuve est de 10 points. Les autres réponses ont été codées en trois catégories:

- erreurs morphologiques (pour l'exemple de l'item 1, il, ils ou elle parle au lieu de elles parlent; pour l'exemple de l'item 2, la ou les mange au lieu de le mange);

- erreurs syntaxiques (omission du pronom: pour l'exemple de l'item 1, parlent au lieu de elles parlent; pour l'exemple de l'item 2, mange au lieu de le mange);

- réponses inappropriées sur le plan discursif (reprise inappropriée du syntagme nominal : pour l'exemple de l'item 1 , les filles discutent au lieu de elles discutent; pour l'exemple de l'item 2, mange le poisson au lieu de le mange).

\section{Résultats}

D'une manière générale, le nombre d'emplois corrects de pronoms par les enfants sourds est nettement inférieur à celui produit par les enfants du groupe contrôle ( $23 \%$, contre $53 \%$ pour le groupe de $2 \mathrm{P}$ et $71 \%$ pour celui de $4 \mathrm{P}$ ).

\section{Modailités orale vs écrite}

Lorsque l'on compare les résultats en modalités écrite et orale, on constate que les enfants sourds produisent davantage de pronoms corrects à l'écrit qu'à l'oral ( $32 \%$ vs $16 \%$, voir Figure 1), alors que chez les enfants entendants, la proportion d'emplois corrects de pronoms est plus élevée dans la tâche orale que dans la tâche écrite (groupe $2 \mathrm{P}: 65 \%$ vs $41 \%$; groupe $4 \mathrm{P}: 78 \%$ vs $64 \%$ ). Le test statistique de Wilcoxon, qui permet de comparer les rangs obtenus par chaque sujet par rapport à son groupe dans les deux modalités, révèle que ces différences de performance sont significatives pour chacun des trois groupes. 


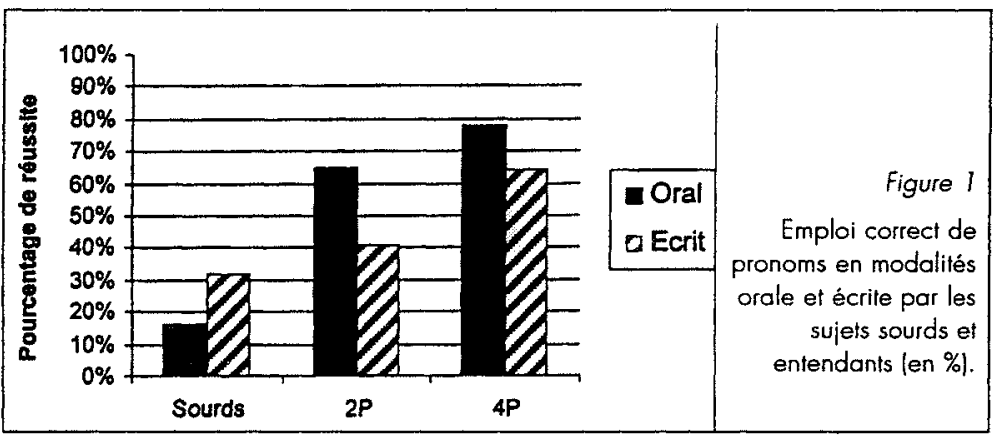

L'analyse par sujet corrobore fortement ces résultats. Dans le groupe contrôle, 29 enfants sur 40 réussissent mieux l'épreuve orale que l'épreuve écrite ( $73 \%$ des sujets) alors que chez les enfants sourds 8 enfants sur 11 réussissent mieux l'épreuve écrite que l'épreuve orale ( $73 \%$ des sujets).

\section{Pronoms sujets vs pronoms objets}

\section{Modalité orale}

Dans la modalité orale, on obtient un pattern similaire pour chaque groupe: le pourcentage de pronoms sujets corrects, bien que légèrement supérieur, reste proche de celui des pronoms objets. Pour les enfants sourds, parmi le peu de pronoms produits, le rapport pronoms sujets corrects/pronoms objets corrects est de deux pour un ( $18 \%$ vs $9 \%$ ) mais la différence demeure statistiquement non significative (voir Figure 2a).

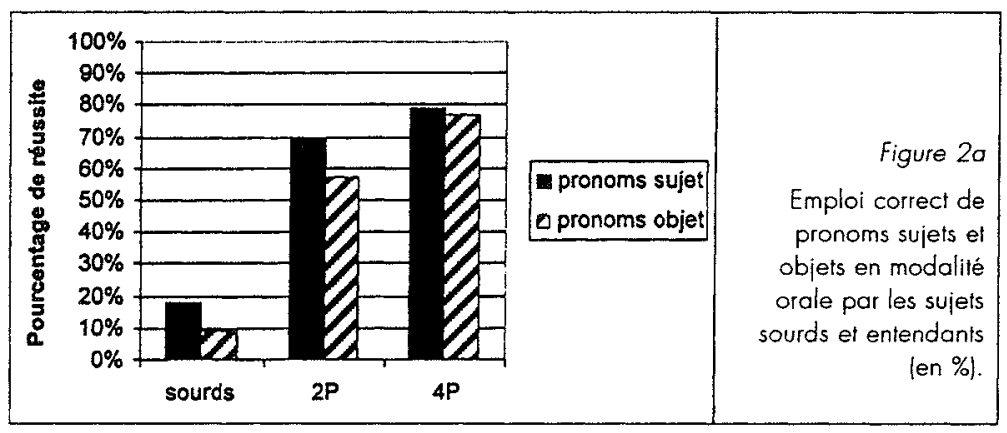




\section{Modalité écrite}

Dans la modalité écrite, les résultats sont au contraire très différents d'un groupe à l'autre (voir Figure 2b). Les sujets contrôle de 4P produisent des pronoms corrects sujets et objets dans des proportions similaires $(68 \% v s 60 \%$; le test de Wilcoxon ne révèle pas de différence significative). Les sujets contrôle de $2 \mathrm{P}$ produisent nettement moins de pronoms corrects sujets que objets ( $34 \%$ de pronoms sujets vs $60 \%$ de pronoms objets; le test de Wilcoxon révèle une différence significative). Quant aux sujets sourds, ils réussissent davantage les items portant sur les pronoms sujets que ceux portant sur les pronoms objets $(47 \%$ vs $9 \%$; le test de Wilcoxon révèle une différence significative). Leur taux de réussite sur les pronoms sujets est même supérieur à celui des $2 \mathrm{P}(47 \%$ contre $34 \%$ ). Ce meilleur résultat des enfants sourds sur les entendants de $2 \mathrm{P}$ concernant le pronom sujet en modalité écrite, ainsi que leur faible taux de réussite concernant le pronom objet dans les deux modalités, seront repris dans la discussion.

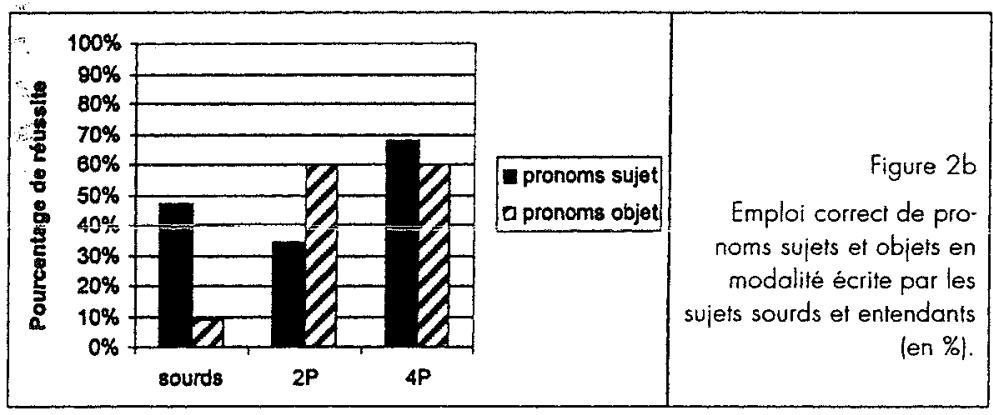

\section{Types d'erreurs}

\section{Modalité orale}

Aussi bien les sujets sourds que les sujets entendants produisent des réponses inappropriées sur le plan discursif, dans des proportions relativement proches $(16 \%$ à $22 \%$, voir Tableau 1). Rappelons que ce type de réponses consiste à reprendre le nom produit par l'expérimentateur alors qu'un pronom s'y référant serait plus approprié dans cette situation. En outre, les sujets sourds produisent des erreurs de type syn- 
taxique (omissions) rares chez les sujets entendants. Notons encore que la moitié des sujets sourds a choisi de recourir à la langue des signes pour répondre à certains items, avec des productions dans la plupart des cas correctes, faisant appel à des procédés anaphoriques spécifiques tels que le pointage ou la prise de rôle difficilement classables dans les catégories prévues pour la langue française ( $26 \%$ du total des réponses). Ce type de réponse a été classé dans la catégorie «Autres», de même qu'une des réponses des sujets du groupe $2 \mathrm{P}$ difficilement interprétable.

\begin{tabular}{llllll}
\hline & Erreurs & & \multicolumn{2}{l}{$\begin{array}{l}\text { Réponses } \\
\text { non attendues }\end{array}$} & $\begin{array}{l}\text { Emplois } \\
\text { corrects }\end{array}$ \\
& Morph. & Syntax. & Disc. & Autres & \\
Sourds & 1 & 34 & 20 & 29 & 16 \\
2P & 4 & 8 & 22 & 1 & 65 \\
$4 \mathrm{P}$ & 1 & 5 & 16 & 0 & 78 \\
\hline
\end{tabular}

Tableau 1 - Types de réponses par catégories (en \%) pour les sujets sourds et entendants en modalité orale, pronoms sujets et objets confondus.

\section{Modalité écrite}

Aussi bien les sujets sourds que les sujets entendants produisent des erreurs syntaxiques et des réponses inappropriées sur le plan discursif, cependant dans des proportions différentes (voir Tableau 2). En outre, les sujets entendants produisent des erreurs morphologiques, surtout les $2 \mathrm{P}$ (19\%) qui sont pratiquement absentes dans le groupe des enfants sourds (4\%). Ainsi, les enfants sourds, lorsqu'ils utilisent des pronoms dans leur réponse, les utilisent pratiquement toujours dans une forme correcte (par exemple elles parlent pour l'item 1), contrairement aux jeunes enfants entendants, qui commettent des erreurs essentiellement sur les marques du pluriel (*elle parles). Le test du chi-carré, qui permet de comparer entre deux groupes la distribution des réponses dans les catégories définies, révèle que ces distributions sont différentes de manière très significative. Ces différences seront discutées plus loin. 


\begin{tabular}{llllll}
\hline & Erreurs & & \multicolumn{2}{l}{$\begin{array}{l}\text { Réponses } \\
\text { non attendues }\end{array}$} & $\begin{array}{l}\text { Emplois } \\
\text { corrects }\end{array}$ \\
& Morph. & Syntax. & Disc. & Autres & \\
Sourds & 4 & 22 & 40 & 2 & 32 \\
2P & 19 & 19 & 17 & 4 & 41 \\
$4 \mathrm{P}$ & 11 & 9 & 15 & 1 & 64 \\
\hline
\end{tabular}

Tableau 2 - Types de réponses par catégories (en \%) pour les sujets sourds et entendants en modalité écrite, pronoms sujets et objets confondus.

\section{Discussion}

Comme nous l'avons supposé, les résultats montrent que, contrairement aux enfants entendants, dont les performances sont meilleures à l'oral qu'à l'écrit, les enfants sourds réussissent mieux l'épreuve écrite que l'épreuve orale. Cela est vrai essentiellement pour les pronoms sujets, pour lesquels nous constatons près de $50 \%$ de réussite. Sur ce plan, les performances des sujets sourds sont supérieures à celles des entendants de $2 \mathrm{P}$. Nous constatons également que les sourds ne font pratiquement pas d'erreurs morphologiques contrairement au groupe contrôle. Ces résultats quantitatifs et qualitatifs suggèrent que les enfants sourds bilingues interrogés construisent leurs connaissances des pronoms écrits sans la référence à l'oral que l'on observe chez les enfants entendants. Ils n'auraient pas à passer par cette étape intermédiaire où les enfants entendants, tout en produisant correctement les pronoms avec les marques morphologiques repérables à l'oral, font de nombreuses erreurs sur les marques propres à l'écrit, donc non audibles. Les enfants entendants doivent en effet intégrer celles-ci au sous-système qu'ils ont déjà construit pour l'oral. Pour les enfants sourds, on peut imaginer d'autres stratégies à l'œuvre, reposant sur les connaissances construites en LSF, leur langue dominante, et sur leur compétence métalinguistique. Leurs connaissances en LSF pourraient les familiariser notamment avec les catégories linguistiques générales telles que nom, pronom, sujet syntaxique. Leurs connaissances métalinguistiques les rendraient sensibles à certaines propriétés de l'écrit, par exemple à la 
relation entre une catégorie comme le pluriel et les marques qui lui sont associées.

Quant aux pronoms objets, s'ils sont bien réussis à l'écrit par les enfants entendants, même les $2 \mathrm{P}$, c'est probablement parce que leurs différentes formes sont bien distinctes à l'oral. Pour les enfants sourds, nos données confirment les observations faites par d'autres chercheurs, et révèlent que ce type de pronom est rarement produit. La connaissance de la LSF n'apporte au sujet que peu d'indications sur ce type d'unité, rarement produite dans cette langue en tant qu'élément séparé et le plus souvent incorporée dans le verbe (par exemple, dans l'énoncé «Le garçon a pêché un poisson et l'ours LE mange», le complément du verbe "manger» sera, en LSF, incorporé dans le signe verbal, en modifiant certains des paramètres de la forme de celui-ci : le point de départ du mouvement sera le point de référence spatial où le signe «poisson» a été produit et la configuration de la main sera adaptée à la forme de l'objet ingéré, en l'occurrence largement ouverte pour représenter la prise du poisson entier). On peut penser que la connaissance des pronoms objets écrits serait acquise grâce à la réflexion métalinguistique à un moment ultérieur du développement comme le suggère un commentaire d'un des sujets sourds les plus âgés, qui propose après-coup de remplacer la construction Article + Nom par un pronom objet «pour faire plus court» (Niederberger, sous presse).

Placé dans des situations de production de pronoms sujets et objets en modalités orale et écrite, le groupe d'enfants sourds bilingues interrogés a montré des difficultés importantes, mais aussi des capacités ciblées sur la production écrite du pronom sujet. Les performances de ces enfants sur cette partie du système pronominal se révèlent meilleures que celles des enfants entendants de $2 \mathrm{P}$. Ce résultat confirme notre hypothèse qui postulait pour ce type de population le développement de capacités en français écrit précédant celui de capacités similaires en français oral et suggère, avec les types d'erreurs observés autant chez les sourds que chez les entendants, un parcours d'apprentissage spécifique aux enfants sourds. Ce chemin différent leur éviterait paradoxalement cer- 
tains problèmes rencontrés par les sujets entendants dans leur approche de l'écrit. En effet, notre étude révèle chez les apprenants entendants des difficultés initiales à intégrer les marques morphologiques spécifiques de la langue écrite au système pronominal construit à partir de leur connaissance de l'oral, difficultés conduisant principalement à des omissions de marques de nombre pratiquement absentes chez les apprenants sourds interrogés. De nouvelles recherches seront nécessaires pour étudier de plus près l'acquisition du pronom objet et pour approfondir le rôle de la connaissance de la LSF et de la compétence métalinguistique dans l'apprentissage de l'écrit chez l'enfant sourd bilingue.

\section{Références bibliographiques}

Chamberlain, C. et MaYberry, R.I. (2000): Theorizing about the relation between American Sign Language and Reading, in C. Chamberlain, J. P. Morford et R. I. Mayberry (dir.), Language acquisition by eye, Mahwah, Lawrence Erlbaum Ass., 221-259.

Dubuisson, C. et BASTIEN, M. (1998) : Que peut-on conclure des recherches portant sur la lecture par les sourds? In C. Dubuisson et D. Daigle (dir.), Lecture, écriture et surdité, Montréal, Éd. Logiques, 73-101.

HAGE, C. (1994): Développement de certains aspects de la morpho-syntaxe chez l'enfant à surdité profonde. Rôle du Langage Parlé Complété, Thèse de doctorat, Université Libre de Bruxelles.

JACQ, G., TULLER, L. et FuET, F. (1999) : Spécificités morphosyntaxiques du français de l'enfant sourd. Une étude comparative, Glossa, 69, 4-14.

Lepot-Froment, C., et Clerebaut, N. (1996): L'enfant sourd: Communication et Langage, Bruxelles, de Boeck et Larcier.

Leybaert, J. et Alegria, J. (1986) : Processus de lecture chez l'enfant sourd. Une approche psycholinguistique, Vivre sourd aujourd'hui... et demain? Tome 3, Communication et surdité, Bruxelles, EDIRSA, 93-116. 
NIEDERBERger, N. (2004): Capacités langagières en Langue des Signes Française et en français écrit chez l'enfant sourd bilingue: quelles relations? Thèse de doctorat en Psychologie, Université de Genève.

Niederberger, N. (sous presse): Rééducation de l'enfant sourd profond. Bilinguisme, in P. Dulguerov et M. Remacle (dir.), Précis d'audiophonologie et de déglutition, Marseille, Editions Solal.

Niederberger, N. et BERTHOUd-PAPANDROPOULOU, I. (2002): The Use of Pronouns in French by Bilingual Deaf Children. A Specific Path towards Mastery?, communication affichée présentée au IXe International Congress for the Study of Child Language, Madison, Wisconsin, USA, 1621 Juillet 2002.

PADDEN, C. (1993): Lessons to Be Learned from the Young Deaf Orthografer, Linguistics and Education, 5, 71-86.

Prinz, P. et STRONG, M. (1998): ASL Proficiency and English Literacy within a Bilingual Deaf Education Model of Instruction, Topics in Language Disorders, 18 (4), 47-60.

TUlLER, L. (2000) : Aspects de la morphosyntaxe du français des sourds, Recherches linguistiques de Vincennes, 29, 143-156. 